\title{
A COMPUTER ARCHITECTURE FOR INTELLIGENT MACHINES
}

\author{
NAGW-1333
}

by

D.R. Lefebvre and G.N. Saridis

\author{
Rensselaer Polytechnic Institute \\ Electrical, Computer, and Systems Engineering \\ Troy, New York 12180-3590
}

December, 1991

CIRSSE REPORT \#108 


\title{
A Computer Architecture for Intelligent Machines
}

\author{
D.R. Lefebvre \\ G.N. Saridis \\ Electrical, Computer, and Systems Engineering Department \\ Rensselaer Polytechnic Institute \\ Troy, New York 12180-3590
}

\begin{abstract}
The Theory of Intelligent Machines proposes a hierarchical organization for the functions of an autonomous robot based on the Principle of Increasing Precision With Decreasing Intelligence. An analytic formulation of this theory using information-theoretic measures of uncertainty for each level of the intelligent machine has been developed in recent years. This paper presents a computer architecture that implements the lower two levels of the intelligent machine. The architecture supports an event-driven programming paradigm that is independent of the underlying computer architecture and operating system. Details of Execution Level controllers for motion and vision systems are addressed, as well as the Petri net transducer software used to implement Coordination Level functions. Extensions to UNIX and VxWorks operating systems which enable the development of a heterogeneous, distributed application are described. A case study illustrates how this computer architecture integrates real-time and higher-level control of manipulator and vision systems.

1 Introduction
\end{abstract}

The Theory of Intelligent Machines, as proposed in [8], describes a hierarchical organization of the functions of an autonomous robot into three levels: an Execution Level containing the hardware and basic control functions, a Coordination Level integrating the capabilities of the intelligent machine across hardware systems, and an Organization Level providing higher-level planning and reasoning capabilities. The nature of the intelligent machine levels are distinctly different with the Execution Level dominated by control functions, the Coordination Level characterized by operations research issues, and the Organization Level employing artificial intelligence techniques. A diagram of the intelligent machine (IM) is shown in Figure 1. In this model, robotic functions and data are organized in the hierarchy according to the principle of Increasing Precision With Decreasing Intelligence [12][9]. Specifically, higher-level reasoning is performed on abstract information, to which actual data is instantiated at run-time. Detailed run-time information is maintained at the lowest Execution Level and only status information is propagated upward through the hierarchy.

Research in the Center for Intelligent Robotic Systems for Space Exploration (CIRSSE) at Rensselaer Polytechnic Institute over the past several years has addressed the analytic formulation of the Theory of Intelligent Machines. An information theoretic approach has been adopted using entropy as a measure of uncertainty (or inversely, information) at each level of the hierarchy. Thus the "intelligent machine problem" is formulated as an optimal control problem at the Execution Level, a linguistic decision making problem [11][16] at the Coordination Level, and a neural net relaxation problem [5][10] at the Organization Level. With the theory in place, CIRSSE began in 1991 to address the issue of implementing the intelligent machine structure. The computer architecture that has been built to support this research is the focus of this paper. 
The paper is organized as follows. Section 2 describes the mapping of the intelligent machine model to robotic hardware and computers. Section 3 presents the CIRSSE Testbed Operating System (CTOS) that was developed to provide a uniform programming interface for the heterogeneous computing environment. Motivation for and general design details of the Execution Level control systems for the manipulator and vision systems are treated in sections 4 and 5 , respectively. Petri net transducer software and development of Coordination Level functions is covered in section 6 . And, section 7 presents a case study that illustrates how this computer architecture integrates real-time and higher-level control of manipulator and vision systems.

\section{Mapping Intelligent Machine Model to Hardware}

The testbed hardware at CIRSSE consists of an 18-DOF manipulator subsystem, a 5 camera vision subsystem, their associated control computers, and several UNIX workstations. The 18-DOF manipulator is built from two 6-DOF PUMA robots (a PUMA 560 and a PUMA 600) mounted on a custom-built robot transporter with two 3-DOF carts. The carts travel along a 12 foot linear track and provide $\pm 45^{\circ}$ of tilt and $\pm 150^{\circ}$ of rotation. Manipulator hardware may be configured as two independent 6 - or 9-DOF robot arms with a large work volume for assembly experiments, or as an 18-DOF system for cooperative two-arm applications. This manipulator subsystem is controlled by a Motion Control System (MCS), developed at CIRSSE, executing on a dedicated VME cage that is interfaced to the Unimation controller Q-bus, thus bypassing the vendor's controller. MCS software was developed to support a wide range of research into control of multiple manipulators, and integration of IM functions.

The vision subsystem has five cameras that may be used individually or pairwise, a laser scanner for pointing or structured light, and a (VME-based) Datacube image processing system. A Vision Services System (VSS) has been developed to control the vision subsystem and to integrate it into the CIRSSE testbed. Like the MCS, the VSS was designed to be a flexible platform for research.

Additional processing capabilities are provided by four UNIX workstations (predominantly Sun 4 and Sparc Stations) attached to the CIRSSE Ethernet backbone which connects all of the testbed subsystems. A schematic diagram of the CIRSSE testbed hardware is shown in Figure 2.

A mapping of intelligent machine functions to the testbed hardware, also shown in Figure 2, assigns Execution Level functions of motion and vision control to the VME cages and higher-level Coordination and Organization functions to the UNIX workstations. The primary rationale for this division is that control functions must execute in a real-time environment while coordination and planning functions execute at a "human time scale" supportable by UNIX. The VxWorks operating system is used on the VME cages, and forms the basis for MCS and VSS. While VxWorks provides an excellent real-time environment for implementation of control functions, it offers only low-level support (UNIX-compatible sockets) for communication between the processors on the VME cage or between the VME cage and other chassis on the network. UNIX has a similarly limited choice of communication methods between chassis (specifically sockets or System $\mathrm{V}$ messages).

Clearly, the intelligent machine organization proposed in Figure 2 is an heterogeneous, distributed application - spanning many processors, using two different operating systems, and dealing with time scales ranging from $5 \mathrm{~ms}$ control loops to path planning performed aperiodically. To cope with these demands. extensions to UNIX and VxWorks were developed that affect a communication layer between the operating system and the application, and produce a uniform programming interface for developing event-driven tasks. These operating system extensions are called the CIRSSE Testbed Operating System (CTOS) and are the subject of the next section. The researcher is now presented with the straight-forward software model of the intelligent machine shown in Figure 3. 


\section{CIRSSE Testbed Operating System}

The motivation for developing the CIRSSE Testbed Operating System (CTOS) was to enable researchers to build distributed applications that access the CIRSSE testbed hardware. A simple, uniform programming interface was desired which would encourage the development of modular functions that could be interchanged - to construct applications from standard components - and moved among processors - to balance loads.

In broad terms, CTOS supports three objectives: 1) distribution of tasks, 2) communication between tasks, and 3) synchronization of tasks and the application. An event-driven environment was implemented in which tasks execute independently and in parallel, and communicate by exchanging messages. The interface to CTOS is through five services; three of which are available on both UNIX and VxIVorks, and two for real-time programming in VxWorks:

- Bootstrap service loads and initializes software modules on all processors involved in the application. The distribution of tasks, including most CTOS system tasks, is specified via configuration files. A task may be moved from one processor to another simply by revising the configuration file and rebooting the application - no recompilation or relinking is required. Additionally, this service broadcasts messages to all tasks to synchronize three startup phases for the distributed application.

- Task identification service assigns a unique identification to every task created in the application and associates it with the symbolic name of the task specified in the configuration file. The task id is used to route messages to the task. Another task's id can be found from its symbolic name through a request to this service.

- Message passing service is the primary communication mechanism between event handler tasks. When CTOS is started sockets are open between all processors on a VME cage, and between all UNIX workstations and VME cages used by the application. However the user need not know where tasks are located, from the users' viewpoint the same message service is used independent of the location of the receiver since the message is essentially addressed via the receiver's symbolic name.

- Synchronization service provides high frequency, low latency execution of real-time software functions on a VME cage. This service is used primarily by the lowest-level functions of MCS such as control algorithms and hardware interfaces.

- Inter-processor blocking service constitutes a second faster-but-limited communication mechanism between processors on a VME cage based on a "distributed semaphore." It is the foundation for the synchronization service.

The central objects of CTOS are messages and event handler tasks; these are key to understanding the system. Messages are 16 byte data structures that contain the task id of receiving and sending tasks, a command indicating the function of the message, a pointer to additional data and its size, and flags to specify message handling options. When messages are sent between chassis (an individual UNIX workstation or a VME cage) the additional message data is transmitted immediately after the message itself; whereas shared memory techniques are used for messages within a chassis. Management of message data is performed by CTOS, and so is transparent to the user. Message handling flags can specify that messages are to be routed at high priority, that the sending task is to wait for a reply from the receiving task, and which task "owns" the data pointed to by the message.

CTOS users write applications as a collection of event handler tasks which, as discussed earlier, may be distributed among many processors, and which execute in parallel. An event handler task, or simply "task", 
is an instantiation of an event handler function. The CTOS message passing service manages a message queue for each event handler task, and calls the event handler function when a message arrives.

All event handler functions have the same general format shown in Figure 4. Specifically, the task id of that instantiation of the function and a pointer to the received message are passed to the function. Typically the function decodes the message command to determine the processing being requested, performs the processing, passes the processed message and any unrecognized message to default processing, and then exits. Because multiple tasks on a single processor may use the same function, these event handler functions must be reentrant. Requesting service from another task is as simple as sending a message as suggested by the example in Figure 5. Figures 4 and 5 illustrate one action of the task identification service mentioned above.

\section{Motion Control System}

One of the more significant development efforts at CIRSSE has been the production of a Motion Control System (MCS). After a review of control systems available from university [3][15][14] and NASA [1] sources it was recommended that a new system be developed in-house, rather than adapting an existing system or contracting for a custom-built system. Desired functionality, available hardware, and CIRSSE's research agenda were the primary factors favoring an in-house development. These positive factors offset concerns over the size of the effort and timing. In total, the specification, design and implementation of CTOS and core MCS functions required approximately 1.6 man-years of work over a 9 month period - roughly evenly split between CTOS and MCS [18].

Figure 6 is a diagram of the organization of MCS. It is designed with a layered structure and has well defined interfaces between layers so that modules can be replaced independently. The lowest-level testbed interface layer contains the channel drivers that effect a hardware-independent interface for the upper layers of MCS. Each robot joint and external sensor of the manipulator subsystem is assigned a slot in the interface; thus presenting an hardware-independent view of the testbed. For example, a 9-DOF manipulator can be configured from 6 DOF of a PUMA plus 3 DOF from the robot transporter. The next layer up is the motion control layer where controller algorithms compute control torques based on current joint data read from the testbed interface layer and desired setpoints supplied by the motion planning layer. Both the testbed interface and motion control layers are composed of synchronous tasks executing at the servo rate, e.g. every $4.5 \mathrm{~ms}$. The role of the motion planning layer is to generate a trajectory for implementation by the motion control layer. Trajectory setpoints are typically calculated at a frequency that is a multiple of the servo frequency. Requests for motion along new path segments arrive at the motion planning layer asynchronously from the client interface layer. The client interface layer presents the primary means of access for users who wish to use the manipulator subsystem without dealing with low-level MCS functions. The Coordination Level of the IM would move the robot via calls to the client interface layer, for example.

To coordinate the operation of the MCS, a state manager spans the layers of the system. The duties of the state manager are 1) to ensure that the components of MCS are present and properly configured; 2) to allocate resources and prevent contention, e.g. two tasks attempting to move the robot in different directions; and 3) to enforce the transitions between states of the manipulator subsystem in an orderly manner. The state manager allows transitions between five states as shown in Figure 7 . The only nonobvious state is the reserved state which is used to ensure that the DOFs of the manipulator have been properly allocated prior to power on. The five MCS states correspond to the three physical states of "power off", "power on, brakes on", and "power on, brakes off." Power is off for cold, reserved, and estopped states; power and brakes are on for the active state; and the brakes are off for motion state.

The functional requirements set for MCS have resulted in a control system with several characteristic, and in some instances unique, features: 
- MCS is a VME-based multiprocessor, real-time control system. Its real-time capabilities are derived from VxWorks, while interprocessor coordination is supplied by CTOS. Services are provided to build distributed functions such as a control algorithm that executes on multiple processors.

- MCS is part of an heterogeneous, distributed, open architecture that enables integration of motion control functions with vision services and the higher level functions of the IM. MCS was codeveloped with CTOS and established many of CTOS's requirements.

- MCS is highly modular with clearly separated motion control operations and easily interchanged modules performing these operations. Essentially, each operation is one or more event handler task.

- Expandability of MCS is ensured by the open architecture of CTOS. As an example, collision detection software developed in a separate CIRSSE research project was easily integrated into MCS to run at real-time - with no modification to the existant MCS core functions.

- MCS configuration is data-driven; e.g. processor load may be balanced by redistributing tasks, or the system can be reconfigured for a different number of processors through changes to a configuration file. This feature is also expected to enhance the portability of MCS.

- Multiple manipulators are supported; for instance, the CIRSSE testbed has 3 manipulators - two PUMAs and the transporter. MCS implements a uniform interface to all the DOFs so that the 18 DOF of CIRSSE's testbed may be partitioned into three 6-DOF, or two 9-DOF, or one 18-DOF manipulator(s).

- MCS has user interfaces to all layers to support a wide range of research. Coupled with its modular design, this feature allows modification of an individual module without disrupting higher or lower layers of MCS. Thus, MCS supports the reseacher developing a new control algorithm as well as those working two-arm cooperative control or the higher levels of the IM.

The above list stresses the architectural aspects of MCS rather than the usual features of a control system such as trajectory generation methods or control laws supported. The reason for this selectivity is to highlight the modular nature of CTOS. To set up an experiment, configuration files are written to specify configuration of manipulator DOFs, choices of control algorithm module and trajectory generation module to be loaded, speeds of servo loops and other synchronous processes, etc. The experimenter can configure MCS to include his own module and use standard modules for the remaining components of the control system, such as hardware drivers or client interface. At present canonical versions of controller and trajectory generator modules are available that implement conventional PID positioning, gravity compensation, and straight-line and joint-interpolated trajectories. Hardware channel drivers, state manager, and client interface modules have been standardized and rarely change.

MCS represents the motion control component of the Execution Level of the IM. The upper IM levels, specifically the Motion Coordinator, access the manipulator subsystem solely through MCS. Consistent with the principle of Increasing Precision With Decreasing Intelligence, the MCS maintains all detailed information regarding the capabilities and current state of the manipulators. This information is accessable to the Motion Coordinator upon request; but ordinarily only status information, e.g. indicating the successful completion of an operation or existance of valid data, is fed back to the coordinator. Additionally, there is no decision making at the Execution Level, MCS simply performs the requested operation and reports results. It is the duty of the Motion Coordinator to decide which operation is appropriate and what corrective actions may be necessary. 


\section{Vision Services System}

The design objectives for the Vision Services System (VSS) were similar to those for MCS: a convenient interface to the testbed vision hardware was desired that could support a range of research and could be integrated into CIRSSE's IM architecture. Hence, like MCS, VSS is built on CTOS.

The organization of VSS, illustrated by Figure 8 , is layered with two levels of centralized managers. At the lowest level are Vision Modules (VM) that call vendor-supplied library routines to directly access the vision subsystem hardware. Due to their direct use of hardware, VM are single-threaded and cannot be called by multiple processes concurrently. Contention for VM caused system crashs in the past, and was one motivation for development of VSS. Therefore, Vision Module Managers (VMM) are provided to control contention for VM and hardware resources. In the present CIRSSE VSS there are two VMM: one managing Datacube image processing hardware and the other managing the laser scanner. The next layer is composed of Tools which combine a call to a corresponding VM with pre- and post-calculations (although some Tools contain only calculations). Tools must request use of a VM through the VMM but typically receive results directly from the VM. Operation of Tools, VMM, and VM are largely hidden from the user.

Conceptually, the user deals with VSS in terms of Services. A vision Service is a high-level operation that is implemented as a series of calls to Tools. A Service is invoked via a $\mathrm{C}$ function call that sends a CTOS message to the Vision Services Manager (VSM) in a manner analogous to accessing the MCS Client Interface. The VSM is the central point of access to VSS, and has the role of routing a vision service request to the appropriate Service. VSM performs another function of initializing the VM that have been loaded for the current application.

Because the user deals with Services and not VM, Tools, etc., a method is provided to ensure that the necessary software modules are loaded based on the list of Services used in the application. From this list of Services, a CTOS configuration file is built that will load the required software on the correct processors and start the manager tasks. Not all available Services are loaded, only those that are needed. This conserves computer memory and saves significant time in initializing VM. Another advantage of a data-driven VSS configuration is that installing new VM and Tools is as easy as modifying a master configuration file where all available VSS Services are specified.

VSS operation is illustrated by the example of processing a SpotFind service request. The request starts as a call by a CTOS task (e.g. the Vision Coordinator) that sends a message to the VSM. VSM examines the message command to determine the type of vision service request and routes the message to the svcSpotFind task. The svcSpotFind task calls four Tools: 1) toolSnap to capture an image, 2) toolImageDisp to display the image on a monitor, 3) toolSpotFind to compute the location of the spot, and 4) toolSpotDisp to display a marker on top of the image on the monitor. Three of these Tools use corresponding VM; specifically vmSnap, vmImageDisp, and vmSpotDisp. To obtain use of a VM, hence the hardware, the Tool sends a request for the VM to the VMM, and will wait until the VM is available if it is in use at the time of the request. When VM processing is complete, results are sent directly back to its Tool. The toolSpotFind Tool performs the calculations to identify the spots in the image and does not call a VM. Finally, the camera coordinates of the spot are returned directly to the requesting task by the svcSpotFind task.

\section{Coordination Level Software}

The role of the Coordination Level in the Theory of Intelligent Machines is to interpret and manage the plans from the Organization Level, add real-time details as appropriate, and communicate instructions to the Execution Level in order to coordinate the operation of the IMI. In addition, the Coordination Level monitors machine performance to provide feedback to refine short-term decision making and to assist replanning when the nominal task plan is not successful [10].

As shown in Figure 1, the Coordination Level of the M is organized into a tree structure, with a Dispatcher 
at the root and multiple Coordinators at the leaves [13]. All communication with the Organization Level is conducted through the Dispatcher; while the Coordinators interface with their corresponding Execution Level functions. Currently the CIRSSE testbed supports four Coordinators that manage manipulator motion, grippers, vision services, and path planning.

Viewed from the bottom up, the Dispatcher is the first component of the IM that deals with the machine as a whole; and thus can coordinate actions across Execution Level functions, e.g. move a manipulator to a position determined by visual sensing. As its name suggests, the Dispatcher's primary function is to receive commands from the Organization Level and to dispatch these commands to the appropriate Coordinator for implementation. Organization Level commands often must be decomposed by the Dispatcher into a sequence of substeps and real-time values must be supplied for the abstract values used by the planner. This description is a simplification, however, as the Dispatcher is called upon to perform a number of other functions such as supporting system-wide communications, scheduling use of resources, detecting and correcting intermediate-level errors, and performing some run-time planning.

A Coordinator can be considered as an expert in applying the deterministic functions of a narrow domain of the Execution Level. For instance, the Motion Coordinator may have several strategies for moving the tool frame of a manipulator into a requested position and orientation (e.g. employing redundant degrees of freedom), and can choose the strategy with the highest probability of success based on current constraints of the environment (obstacles) and time goals. While a nominal approach may be recommended by the organization-level planner, it is the Coordinator's responsibility to reliably accomplish the requested task in real time. Errors are handled first by the Coordinator, and are passed up to the Dispatcher only when a local strategy is not adequate to resolve the condition. In some instances the Dispatcher must turn over error resolution to the Organization Level where operations may be replanned. Feedback is also provided through the Coordinators in order to update knowledge bases and improve decision making of the IM [16].

CIRSSE have employed a linguistic decision schemata approach [11] to model the Coordination Level. In particular, Petri Net Transducers (PNT) have been proposed [1i] as a means to translate an Organization Level command language into a sequence of Coordination Level subtasks.

Petri nets (PN) are tools for modeling the dynamic behavior of discrete event systems that promise to be a useful method for both decomposing Organization Level commands and for real-time control of the IM. Ordinary PN are directed graphs with two types of nodes called places and transitions, which are connected by arcs [7]. Places may contain tokens that indicate the state of the PN. Tokens are moved between places by the firing of a transition. In Generalized Stochastic Petri Nets (GSPN) [4] transitions may require a deterministic amount of time to fire. For the PN software discussed below a two-phase transition firing rule is implemented: 1) transitions are enabled when there are tokens in all input places of the transition, these tokens are removed when the transition fires; 2) firing the transition causes an action, that may result in a time delay; and 3) once the action is complete, token are added to the transition's output places. PN are valuable for simulating concurrent systems because the PN structure can be analyzed for desirable properties such as boundness and deadlock-free operation [6].

PNT are an extension of GSPN in which transitions optionally have an additional enabling condition read from an input tape composed of elements of the input alphabet. This addition permits construction of nets with several parallel (contending) paths that are resolved by the value of the input tape element. Two other extensions have been implemented in the PNT software to allow integration of PNT into CIRSSE's IM architecture: 1) when a transition fires either a local function is called or a CTOS message is sent out to invoke an action, output places are not marked until the action confirms completion; and 2) another enabling condition has been added as a call to an enabling function in order to implement deterministic and conditional "choice among alternatives [2]." As in the case of ordinary PN, when a PNT is used to model the Dispatcher or a Coordinator, the set of places represents the IM's state, and the transitions represent 
events which change machine state. The Dispatcher is a reconfigurable PNT defined by the Organization Level, while the Coordinators have a fixed structure.

A Coordination Level PNT, i.e. the Dispatcher or a Coordinator, may be composed of many subnets that execute concurrently and are distributed among several workstations and VME cages. This heterogeneous, distributed organization of the PNT is made possible by using CTOS to communicate between the subnets. In the example shown in Figure 9 subnet PNET-A is connected to subnet PNET-B by designating place 1 of PNET-B as a remote output place of PNET-A's transition t1; while PNET-B transition t2 outputs to PNET-A place $\mathrm{p} 2$. When transition $\mathrm{t} 1$ fires, a message is sent from task PNET-A to task PNET-B indicating that $\mathrm{a}$ token has been added to place $\mathrm{p} 1$; and tokens are also written to any local output places ( $\mathrm{p} 3$ in this case). Messages may also be used to invoke an action when a transition fires. For instance, the figure shows PNET-B sending a message to a CTOS task when transition $\mathrm{t} 3$ fires. The destination CTOS task may reside on any computer connected to the CIRSSE Ethernet. With this architecture, a Coordination Level PNT, say the Motion Coordinator, may be implemented on a UNIX workstation yet easily communicate with an Execution Level function running on a VME cage, such as the MCS.

In addition to allowing the composition of a PNT as an assembly of subnets, the CTOS architecture supports flexible displays of PNT execution. Figure 9 shows an example with two subnets attached to three displays. Two of the displays, DISP-A and DISP-B, have been configured to show their corresponding subnets. The remaining display, namely DISP-C , shows a subset of places and transitions from two different subnets. This capability of many-to-many connections between subnets and displays enables the construction of overview displays that summarize the operation and state of a complex PNT spanning many subnets. Operation of the Dispatcher and Coordinators can be monitored by means of these overview displays.

Underlying the decomposition and sequencing of commands performed by the Dispatcher and Coordinators is the flow of data required to implement these commands. To support this flow of data the Coordination Level employs a Data Object Manager (DOM) task to maintain globally available data. The DOM's role is to receive data produced by Coordination Level and Execution Level functions, and to later retrieve this data on demand. To coordinate the generation and use of data, each data object stored in the DOM is associated with a place in a PNT which represents the existance and validity state of the object. The case study discussed below will illustrate the interaction of PNT and DOM.

\section{Case Study}

As a case study the M structures needed to implement camera calibration are examined. A simple algorithm is implemented in which a light source mounted on the manipulator's wrist is moved and its position determined by ceiling-mounted stereo cameras. These steps are repeated for a fixed number of predetermined manipulator positions, and then camera calibration parameters are computed from the stored positions and camera coordinates. The entire process is started by a single calibrate_camera command from the Organization Level to the Coordination Level Dispatcher. Therefore, operation of the Organization Level is omitted from this case study.

Dispatcher

The portion of the Dispatcher PNT that translates the calibrate_camera command is presented in Figure 10. The arrival of the calibrate_camera input tape command enables the init_calib transition which fires when its input marking is satisfied, specifically when the robot and camera are available. Firing the init_calib transition causes a local function to be called that prepares for camera calibration, i.e. turns on the light source and opens the file containing predefined manipulator positions, and that initializes the loop variable which will be checked later by the loop_again and loop_done transitions. Execution of the PNT continues as the Dispatcher decrements the loop variable, reads the next manipulator position from the input file, and then moves the robot to this position. When the move_robot transition fires, the Dispatcher 
instructs the Motion Coordinator to perform this action; this can be done in one of several ways: 1) a local function call may be made to procedural code effecting the action, 2) a tape command may be sent to the Motion Coordinator PNT, or 3) a coordination structure as described in [16] may be employed in which an exchange of tokens coordinates the action of Dispatcher and Coordinator. (The last method is used for the case study.) Independent of the method used, the output places of the move_robot transition are not marked until completion of the requested move is acknowledged by the Motion Coordinator.

At this point two parallel paths of the Dispatcher PNT are followed in order to find the coordinates of the light source and the actual position achieved by the robot. Along one path the Dispatcher interacts with the Vision Coordinator and along the other it interacts with the Motion Coordinator. Since the Coordinators and their associated Execution Level subsystems are independent, these actions can truely be performed concurrently. This example clearly illustrates the utility of the PNT for specifying parallel activities.

Completion of the parallel operations discussed in the previous paragraph is indicated by the marking of both spot_done and pos_done places. This marking enables the two contending transitions loop_again and loop_done. These two transitions share the same enabling function, which examines the loop variable initialized by transition init_calib and returns TRUE or FALSE indicating that it is non-zero. However, the enabling function is negated for transition loop_done so only one of the two transitions will fire. This mechanism allows the construction of a deterministic iterative loop similar to a $\mathrm{C}$ language for loop. A conditional test may be used as an enabling function to obtain a structure similar to a vhilo loop. (Recent Petri net theory [2] has shown how this restricted kind of nondeterministic behavior may be analyzed by decomposing the net into deterministic marked graph components. Extention of this theory to PNT is actively being pursued.)

If transition loop_again fires, a token is output to place loop_ready and the Dispatcher repeats the steps for another calibration point. If transition loop_done fires, processing of the calibrate_camera command preceeds with computation the camera calibration parameters and then release of the resources used. Finally, the Dispatcher acknowledges completion of calibrate_camera to the Organization Level.

Vision Coordinator

The firing of a Dispatcher transition commonly results in a request for an action by a Coordinator. To ensure that the coordinator services only one request at a time and that control returns to the originator of the request, a coordination structure may be used to connect the Dispatcher PNT and the Coordinator net. Figure 11 shows an expansion of the Dispatcher find_spot transition to include the coordination structure and, in this case simple, processing of the command at the Vision Coordinator. The timed find_spot transition has been modelled by the two instantaneous transitions start_find_spot and end_find_spot plus the find_spot_active place.

The coordination structure consists of four places used to control access to the Coordinator. In order for transition start_find_spot to fire it must acquire the token from the SI input semaphore. It may then output its request and initiate Coordinator processing by marking the $I$ input place. The marked request place (or alternatively a PNT tape command may be used) ensures than only one path will be enabled in the Coordinator. When processing of the request is complete the VC_done place will be marked; and then the $\mathbf{t}_{f}$ transition restores the input semaphore and returns control to the requesting subnet. Output and output semaphore places, $O$ and SO respectively, are employed to control this exit. Many Dispatcher transitions may by connected to a Coordinator PNT but only one at a time will be granted access by virtue of the coordination structure.

In the study case, find_spot is the only Vision Coordinator service that is required. However, Figure 11 illustrates how additional Vision Coordinator operations would be included. The doffind_spot transition itself is implemented as a local function call that exchanges messages with the VSS to acquire the requested 
information and then stores it with the DOM as discussed in the next subsection.

\section{Motion Coordinator}

The case study requires that the Motion Coordinator service move_robot and find_pos requests from the Dispatcher. The portion of the Motion Coordinator that performs the move_robot operation is presented in Figure 12. The coordination structure and expansion of the Dispatcher move_robot transition is not included.

When a move operation is requested a path may have been previously specified. The Motion Coordinator is designed to accommodate this preplanned path or to generate a path immediately prior to the move. Transitions validate_path and plan_path are another example of choice among alternatives that is resolved through a PNT enabling function (the same function asserted and negated). For the study case the path must be planned, so transition plan_path will fire. This invokes a call to a path planning algorithm that retrieves the manipulator's current position and the destination position from the DOM and later stores the path specification with the DOM. Note that the destination data object was initialized by the earlier read_next_pos Dispatcher transition. The presence of a valid path specification data object in DOM is indicated by marking the path_valid place. This marking also enables the move_manip transition.

Firing the move_manip transition results in a call to a local function that prepares a request message to MCS. It obtains the input arguments for the MCS message from the DOM. MCS acknowledges completion of the manipulator move operation to the local function which then updates the current position data object and completes the movemanip transition. This example illustrates use of the DOM to exchange data objects between PNT transition operations, which are separated temporally and distributed among various processors, and use of PNT places to maintain state information of the existance or validity of data objects in the DOM.

\section{Summary}

The computer architecture of an intelligent machine is likely to be heterogeneous and distributed - reflecting the organization of the IM itself. At CIRSSE, the needs of IM researchers have been met through the development of high-level interfaces (MCS \& VSS) to Execution Level hardware, PNT software for affecting Coordination Level integration, and a means (CTOS) to build distributed applications spanning a heterogeneous computing environment. Using these tools the lower two levels of the IM have been implemented, and the foundation laid for continuation of this work to the IM reasoning functions of the Organization Level.

\section{Acknowledgement}

The M computer architecture at CIRSSE is the product of the efforts of a large number of talented people; in particular, Kevin Holt, Keith Nicewarner, Russ Noseworthy, Lance Page, Joe Peck, Art Ryan, Sanjeev Seereeram, Dave Swift, Lee Wilfinger. and the MCS Design Team - Keith Fieldhouse, Don Lefebvre, Steve Murphy, and Jim Watson. Funding for this work was provided by NASA under Grant NAGW-1333.

\section{References}

[1] J.S. Albus, H.G. McCain and R. Lumia, "NASA/NBS Standard Reference Model for Telerobotic Control System Architecture (NASREM)," NIST Tech. Note 1235, National Institute of Standards and Technology, April 1989.

[2] P. Freedman, "Time, Petri Nets, and Robotics," IEEE Tran. Robotics Automat., vol. 7, no. 4, pp. 417-435, Aug 1991. 
[3] J. Lloyd, M. Parker and R. McClain, "Extending the RCCL Programming Environment to Multiple Robots and Processors," Proc. IEEE Int. Conf. Robotics Automat. (Philadelphia, PA), pp. 465-469, April 1988.

[4] A. Marsan, "Stochastic Petri Nets: An Elementary Introduction," Advances in Petri Nets 1989 (G. Rozenberg, ed.), pp. 1-29, Springer-Verlag, 1989.

[5] M.C. Moed and G.N. Saridis, "A Boltzmann Machine for the Organization of Intelligent Machines," IEEE Tran. Sys., Man and Cyber. vol. 20, 1990.

[6] T. Murata, "Petri Nets: Properties, Analysis and Applications," Proceedings of the IEEE vol. 77 , no. 4, pp. 541-580, 1989.

[7] J.L. Peterson, Petri Net Theory and the Modeling of Systems, Prentice-Hall, 1981.

[8] G.N. Saridis, "Toward the Realization of Intelligent Controls," Proc. IEEE, vol. 67, no. 8, 1979.

[9] G.N. Saridis, "Intelligent Machines: Distributed vs. Hierarchical Intelligence," Proc. IFAC/IMAC Int. Symp. on Distrib. Intelligence Systems (Varna, Bulgaria), pp. 34-39, 1988.

[10] G.N. Saridis, "Architectures for Intelligent Machines," CIRSSE Report 96, Rensselaer Polytechnic Institute, Troy, N.Y., Aug 1991.

[11] G.N. Saridis and J.H. Graham, "Linguistic Decision Schemata for Intelligent Robots," IFAC J. Automatica, vol. 20, pp. 121-126, 1984.

[12] G.N. Saridis and H.E. Stephanou, "A Hierarchical Approach to the Control of A Prosthetic Arm," IEEE Trans. Sys., Man and Cyber., vol. 7, pp. 407-420, 1977.

[13] G.N. Saridis and K.P. Valavanis, K.P. "Analytical Design of Intelligent Machines," IFAC J. Automatica, vol. 24, no. 2, pp. 123-133, 1988.

[14] D.B. Stewart, D.E. Schmitz and P.K. Khosla, "Implementing Real-Time Robotic Systems Using CHIMERA II," Proc. IEEE Int. Conf. on Robotics Automat. (Cincinnati, Ohio), May 1990.

[15] A. Topper, L. Daneshmend and V. Hayward, "A Computing Architecture for a Multiple Robot Controller - KALI Project," 5th CASI Conf. on Astronautics, Nov. 1988.

[16] F. Wang and G.N. Saridis, "A Coordination Theory for Intelligent Machines," IFAC J. Automatica, vol. 26, pp. 833-844, 1990.

[17] F. Wang and G.N. Saridis, "A Formal Model for Coordination of Intelligent Machines using Petri Nets," Proc 3rd IEEE Int. Intell. Control Symp. (Arlington, VA), 1988.

[18] J.F. Watson, et. al., "Testbed for Cooperative Robotic Manipulators," Intelligent Robotic Systems For Space Exploration, Kluwer Academic Press, chap. 1, 1991. 


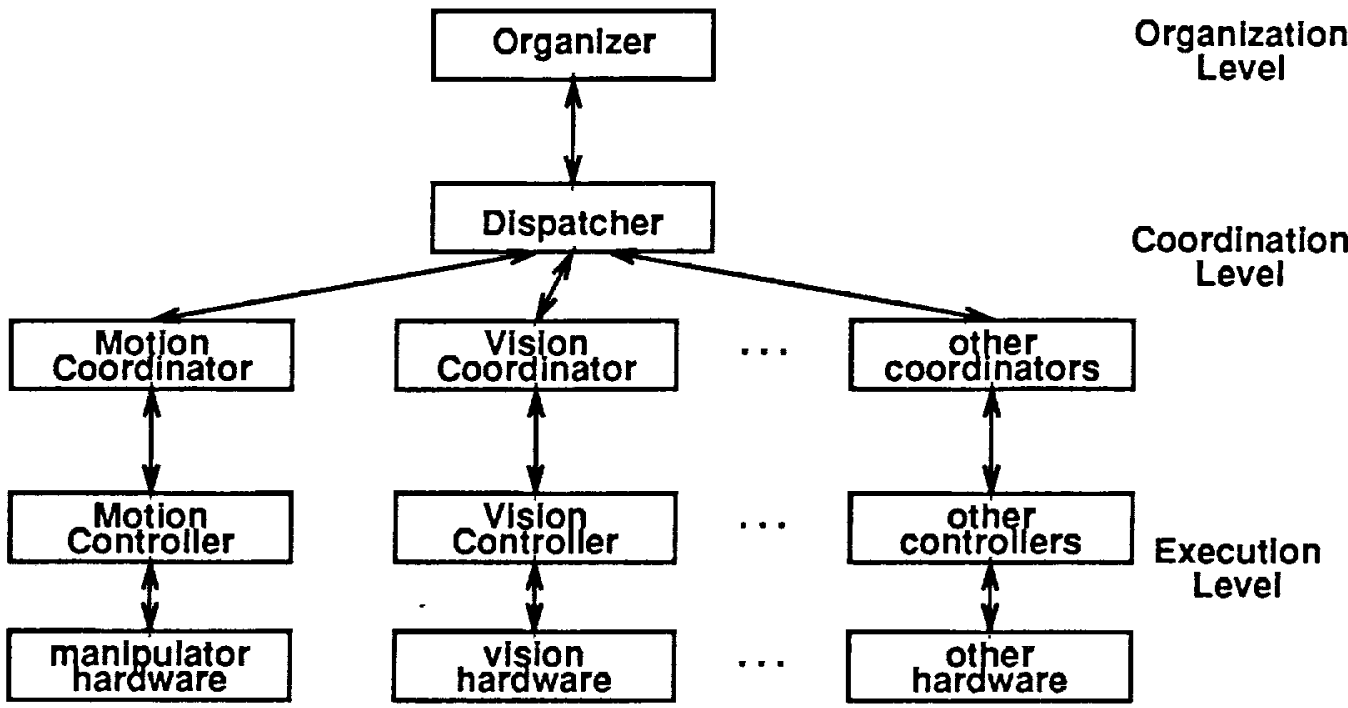

Figure 1: Logical Model of Intelligent Machine

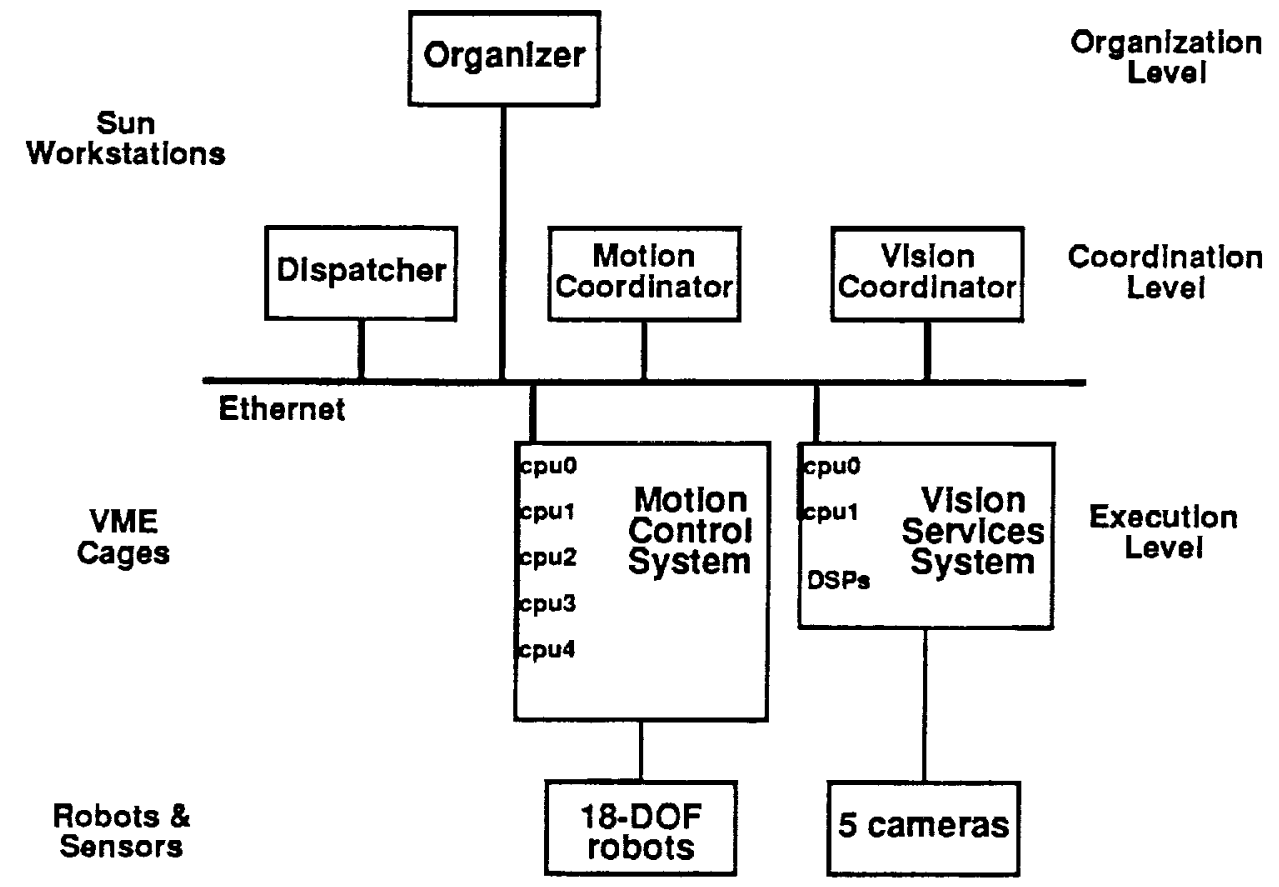

Figure 2: IM Functions Mapped to Testbed Hardware 


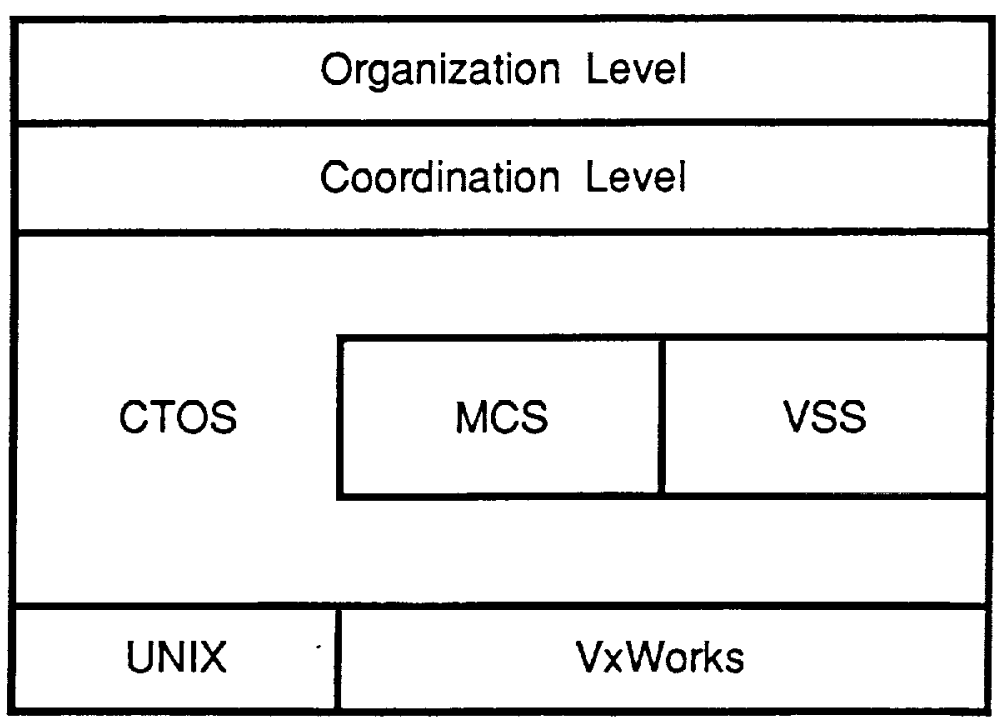

Figure 3: Software Model of Intelligent Machine

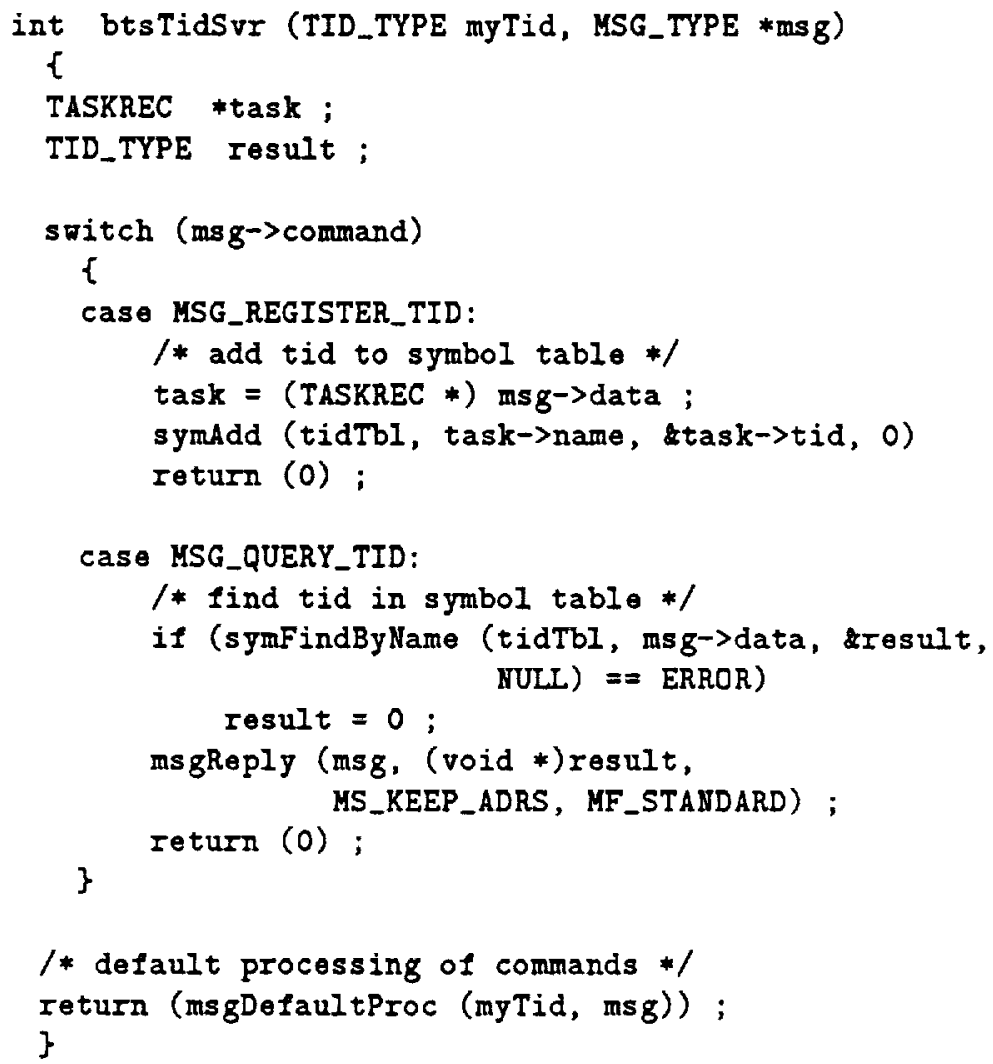

Figure 4: Example Event Handler Function 


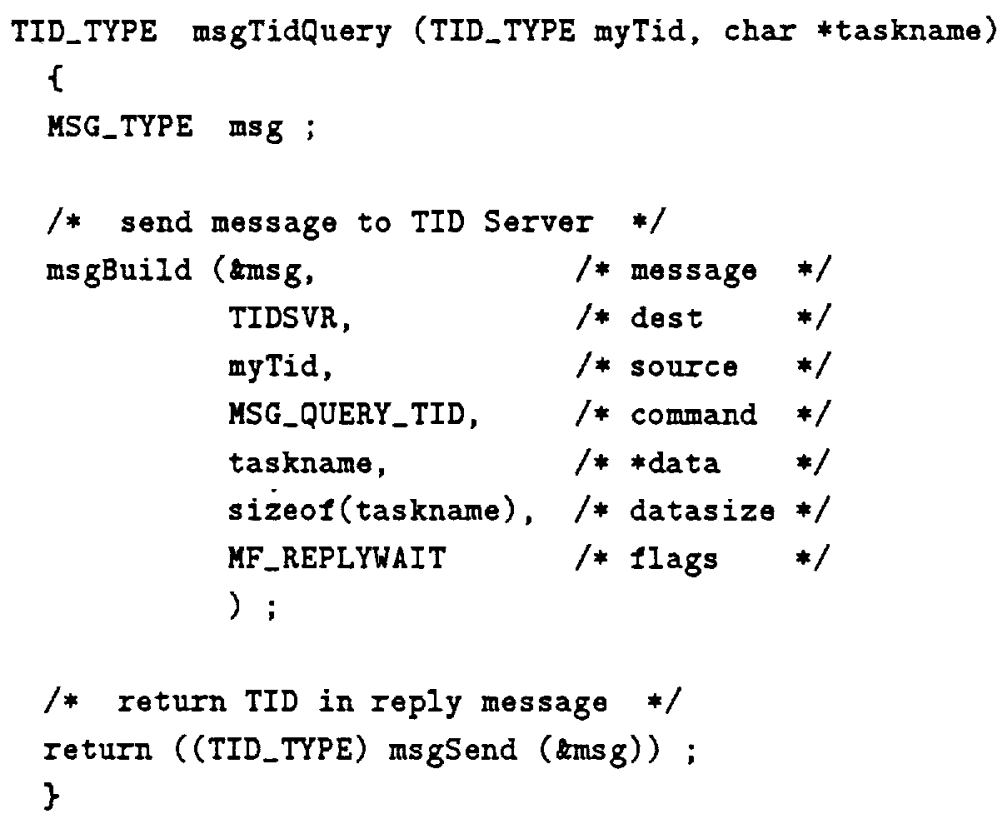

Figure 5: Example Request to Task

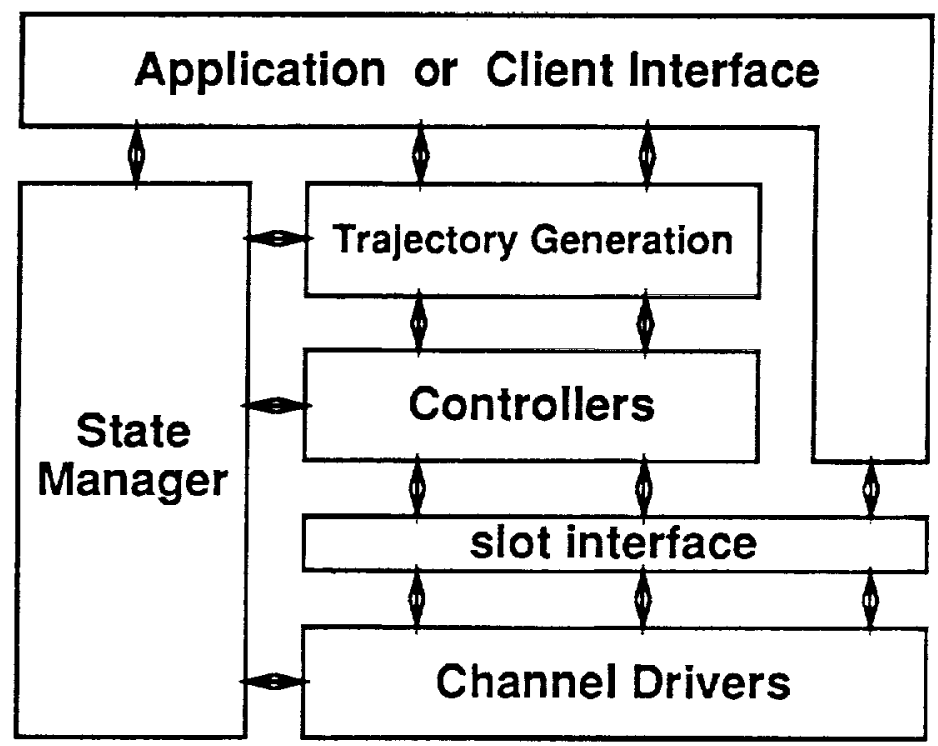

Client Interface Layer

Motion Planning Layer

Motion Control Layer

Testbed Interface Layer

Figure 6: Motion Control System Organization 
Figure 7: MCS State Diagram

(power off)
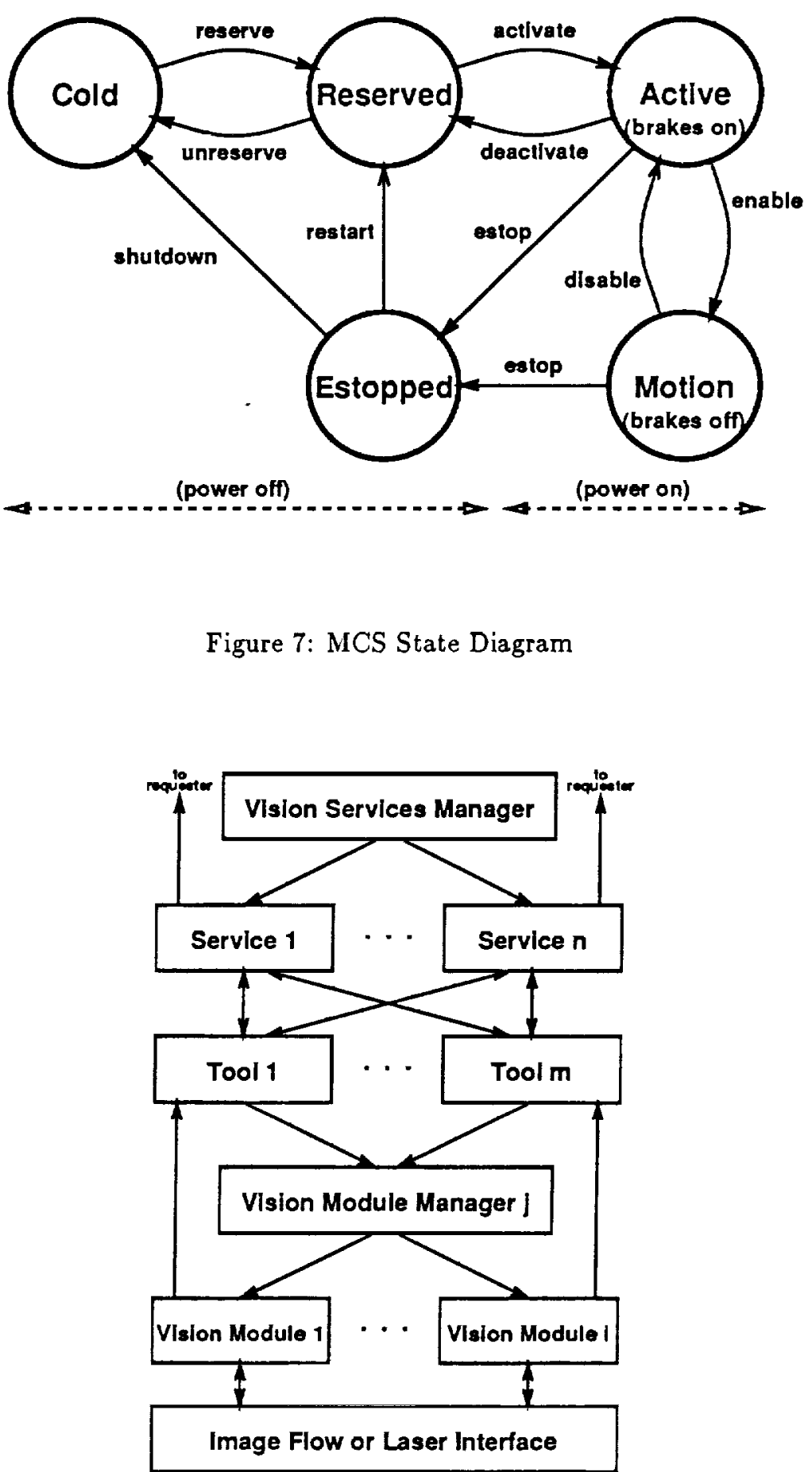

Figure 8: Vision Services System Organization 

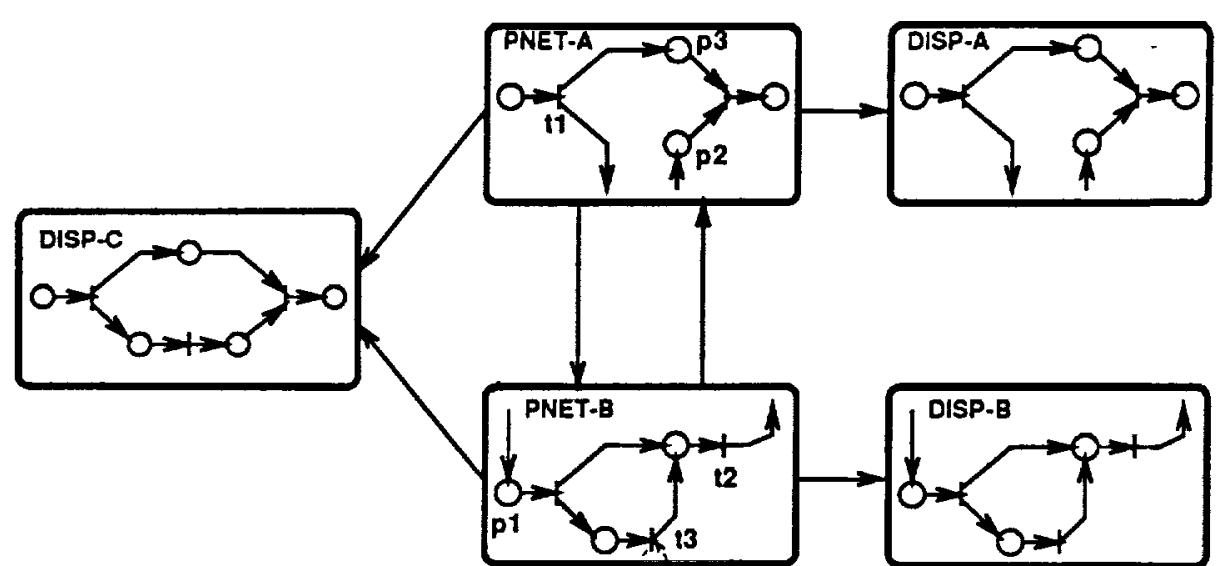

Figure 9: Distributed Petri Net Transducer

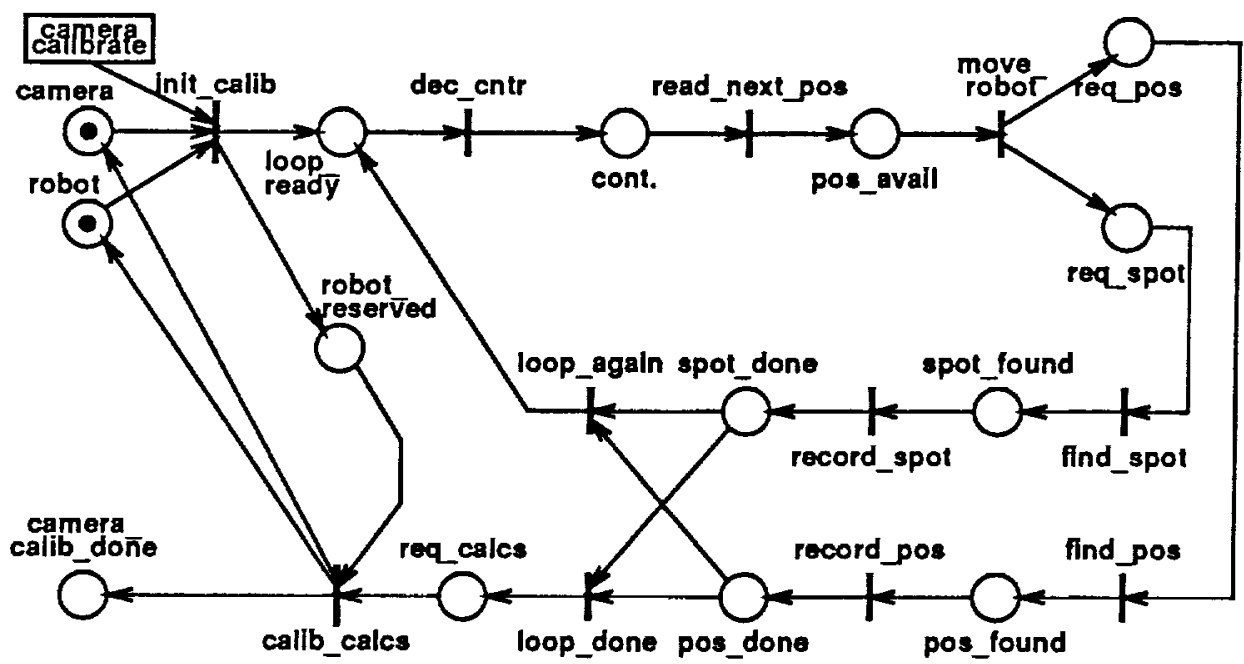




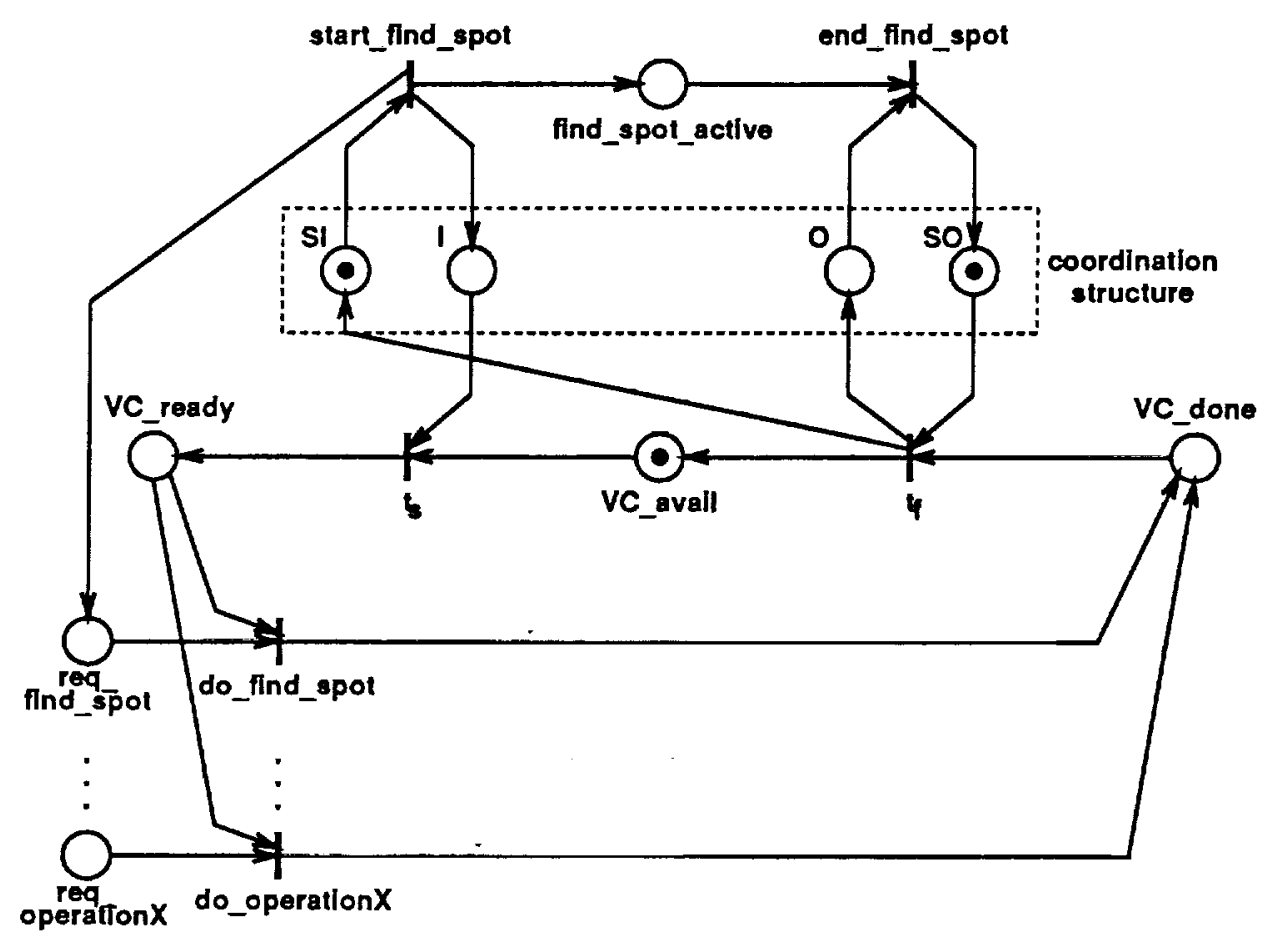

Figure 11: Case Study Vision Coordinator

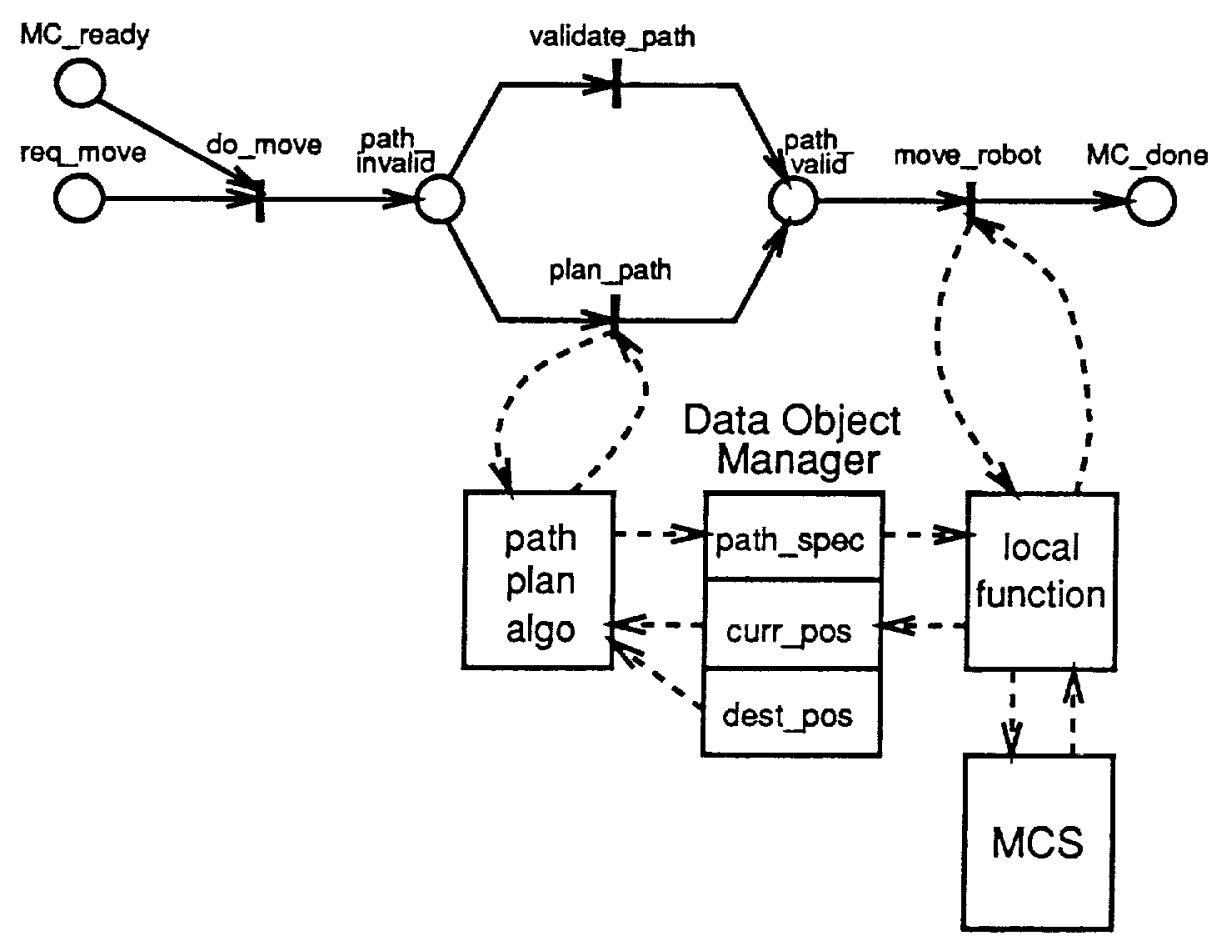

Figure 12: Move Subnet of Motion Coordinator 